\title{
APTITUD COMBINATORIA GENERAL Y CORRELACIONES FENOTÍPICAS ENTRE LÍNEAS Y MESTIZOS DE MAÍZ1
}

\author{
José Luis Ramírez², José Ron², Jesús Sánchez², Abraham Carcía², José Maya ${ }^{2}$
}

\begin{abstract}
RESUMEN
Aptitud combinatoria general y correlaciones fenotipicas entre líneas y mestizos de maíz. El presente trabajo tuvo como objetivos: probar la estrategia de seleccionar líneas utilizando la prueba de aptitud combinatoria general, en forma tardía, y el comportamiento per se de dos grupos de líneas con niveles de endogamia de $\mathrm{S}_{4}$ a $\mathrm{S}_{7}$. Además, estimar las correlaciones fenotípicas entre el rendimiento de grano y otros caracteres agronómicos en líneas y en mestizos, y entre líneas y sus mestizos, para varios caracteres de importancia económica. Se formaron dos grupos de mestizos, uno de ciclo intermedio y otro tardío, utilizándose como probador común la variedad V-385 C3. Simultáneamente, se multiplicaron las líneas. Las líneas per se y los mestizos se sembraron en Zapopan y Tlajomulco, Jalisco. México. El uso de la prueba tardía ( $\mathrm{S}_{3}$ o $\mathrm{S}_{4}$ en adelante), la evaluación simultánea de líneas per se y el método gráfico desarrollado, fue una estrategia importante para seleccionar líneas con alta aptitud combinatoria general y calidad agronómica. Además, fue útil para reducir el número de líneas a evaluar y en orientar anticipadamente el tipo de híbrido a formar. La no significancia y similitud del coeficiente de correlación entre el rendimiento de grano de las líneas per se y los mestizos, en ambos grupos de líneas ( $\mathrm{r}$ $=0,21$ y $\mathrm{r}=0,20$ para líneas intermedias y tardías, respectivamente), confirma que no es del todo confiable seleccionar líneas con base en el rendimiento de sus mestizos, lo cual justifica la estrategia de realizar ambas evaluaciones.
\end{abstract}

\begin{abstract}
General combining ability and phenotypic correlations between corn lines and hybrids. The objectives of this research were: (a) to evaluate the strategy of line selection using the general combining ability test (delayed form), and the performance per se of groups of lines with endogamy levels between $S_{4}$ and $S_{7}$. (b) to estimate phenotypic correlation between grain yield and other agronomical traits in lines and hybrids, and between lines and their hybrids, for several economically important factors. Two groups of hybrids were formed, one intermediate and one late cycle. The V-385 C3 was used as the common testero The lines were simultaneously multiplied. The lines themselves and the hybrids were planted in Zapo pan and Tlajomulco, Jalisco, Mexico. Use of late testing $\left(\mathrm{S}_{3}\right.$ or $\mathrm{S}_{4}$ and later), simultaneous evaluation of the lines themselves, and the graphic method developed were important strategies for selecting lines with a high general combining ability and agronomic quality. The study was also use fuI for reducing the number of lines to evaluate, and for selecting in advance the best type of hybrid to produce. Phenotypic correlation for grain yield between lines and test crosses was similar in the two lines $(r=0.21$ and $r=0.20$ for intermediate and late lines, respectively), and not significant. This confirms that selecting lines using only the grain yield of their test crosses is not a completely reliable procedure, and justifies the strategy of performing both evaluations.
\end{abstract}

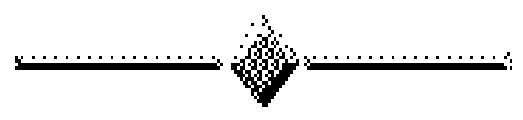

\section{INTRODUCCIÓN}

La obtención de líneas endogámicas con buena capacidad de combinación y alta calidad agronómica que maximice la redituabilidad en la producción de semillas, es la meta de los mejoradores de maíz en la formación de variedades mejoradas para uso comercial. Sin embargo, la integración de estos dos componentes en la selección de líneas se dificulta por el costo elevado en la evaluación de líneas per se y en cruzas de prueba, para medir su calidad agronómica y su capacidad de combinación, respectivamente.

Jenkins (1935) y Sprague (1946), diseñaron la prueba temprana para evaluar la aptitud combinatoria general (ACG) de las líneas en las primeras etapas de

\footnotetext{
1 Este trabajo fue parcialmente financiado por el Consejo Nacional de Ciencia y Tecnología, a través del Proyecto 1588 PB. México.

2 Programa de Mejoramiento genético de maíz del Centro de Investigaciones del Pacífico Centro. Apdo. Postal №10, 45640 Tlajomulco, Jal. Méx. Tels. (377) 2-4051 y Fax. (377) 2-4176.
} 
autofecundación; metodología que pennite eliminar al menos $50 \%$ de las líneas de baja ACG, reduciendo los costos de evaluación de las líneas en las generaciones subsiguientes. Los mismos autores encontraron que la ACG de las líneas quedaba definida en las primeras etapas de autofecundación, lo que posterionnente fue corroborado por Hallauer y López-Pérez (1979), y Bernardo (1992). La aptitud combinatoria general en las líneas es importante porque es infonnativa de la capacidad de la línea en combinación con otros progenitores y está relacionada con su valor aditivo (Sprague y Tatum, 1942).

Dado que la implementación de la prueba temprana de ACG involucra trabajos de polinización y la elección de un probador para la formación de mestizos (línea x probador), se ha intentado simplificarla realizando sólo la evaluación de líneas per se (en $\mathrm{S}_{1}$ ó $\mathrm{S}_{2}$ ), tomando como base, que en resultados encontrados en el mejoramiento poblacionalla respuesta a la selección fue similar utilizando los mestizos y las líneas SI como unidades de selección (Lonnquist, 1968; Márquez, 1985); y en algunos de los sintéticos seleccionados con líneas per se y mestizos, la capacidad de combinación fue igual estadísticamente (Horner, et al., 1969). Sin embargo, cuando se ha comparado la selección de líneas per se versus mestizos, los resultados no han sido consistentes en la discriminación de las líneas, debido, entre otras cosas, a que las líneas tienen mayor interacción genotipo-ambiente que los mestizos (Luna et al., 1973; Galarza et al., 1973, González et al., 1990).

Otra opción seguida por los mejoradores para conjuntar ambas, la buena capacidad de combinación y los caracteres agronómicos deseables, es realizar la prueba tardía, la cual consiste en seleccionar para caracteres agronómicos durante las primeras tres o cuatro generaciones de autofecundación y posterionnente las líneas seleccionadas son cruzadas con un probador para evaluar su capacidad de combinación. Al respecto Bauman (1981) informó, que en los Estados Unidos de América, $0 \%$ de los mejoradores evaluaron en mestizos las líneas en $\mathrm{S}_{1}, 18 \%$ en $\mathrm{S}_{2}, 33 \%$ en $\mathrm{S}_{3}, 27 \%$ en $\mathrm{S}_{4} \mathrm{y}$ $22 \%$ de $\mathrm{S}_{5}$ en adelante, lo que demuestra la preferencia de los mejoradores a realizar la prueba tardía.

Las correlaciones fenotípicas entre el rendimiento de grano de líneas per se y el de sus mestizos no han sido consistentes, Luna et al. (1973) encontraron valores del orden de 0,16 y González et al. (1990) del 0,68. Hallauer (1990) presentó resultados de varios investigadores que confirman esta inconsistencia. No obstante, se ha señalado que una selección efectiva para caracteres deseados en los híbridos puede hacerse en líneas autofecundadas, debido a que los caracteres de vigor en líneas están asociados con el incremento de la productividad de los híbrido s (Jenkins, 1978).
El presente trabajo tuvo como objetivo: probar la estrategia de seleccionar líneas utilizando la prueba de ACG, en fonna tardía, y el comportamiento per se de dos grupos de líneas con niveles de endogamia de $\mathrm{S}_{4}$ a $\mathrm{S}_{7}$. Además, estimar las correlaciones fenotípicas entre el rendimiento de grano y otros caracteres agronómico s en líneas y en mestizos, entre líneas y sus mestizos, para varios caracteres de importancia económica.

\section{MATERIALES Y MÉTODOS}

En el ciclo de otoño-invierno 1993/94, en el Campo Experimental Costa de Jalisco, ubicado en La Huerta, Ja1. México, se formaron dos grupos de mestizos y simultáneamente se multiplicó la semilla de las líneas mediante autofecundación. El primer grupo se integró con 83 líneas tardías derivadas de cuatro poblaciones: Sintético Tardío, integrado con seis líneas élite tardías, (70 líneas), ETO $\left(\mathrm{S}_{2}\right) \mathrm{C}_{1}$ (seis líneas), Pob. 347 (tres líneas) y Pob. 345 (cuatro líneas). El segundo grupo estuvo integrado con 54 líneas de ciclo intennedio derivadas de las poblaciones siguientes: Sintético Intennedio, formado con siete líneas élite intermedias, (doce líneas), BD2 $\left[\mathrm{S}_{1}, \mathrm{~S}_{2}(\mathrm{R})\right] \mathrm{C}_{2}$ (26 líneas), \{[Pool 19 x LB x $\left.\left.\left(\mathrm{LB}\left(\mathrm{S}_{1}\right)\right)\right]^{3}\right\}$ CI (nueve líneas) y siete líneas procedentes del Centro Internacional de Mejoramiento de Maíz y Trigo (CIMMYT). Los niveles de endogamia de las líneas en los dos grupos osciló de $\mathrm{S}_{4}$ a $\mathrm{S}_{7}$ y el probador para ambas grupos fue la variedad de polinización libre $\mathrm{V}-385 \mathrm{C}_{3}$, la cual fue integrada con germoplasma adaptado, no está emparentado con las líneas en los dos grupos y tiene baja calidad de raíz y tallo.

En el ciclo de primavera-verano 1994, los mestizos y las líneas de cada grupo fueron evaluados en ensayos repetidos en dos localidades del centro de Jalisco; Zapopan y Tlajomulco, con una altitud aproximada de 1,590 y 1,550 metros sobre el nivel del mar, respectivamente (García, 1973). Los mestizos y líneas tardías se evaluaron utilizando el diseño experimentallatice simple 10 x 10 y los intennedios en latice simple 8 x 8 . En Zapopan, el tamaño de parcela para los mestizos fue de dos surcos de 5,0 m espaciados a $0,80 \mathrm{~m}$ con 25 plantas cada uno; para las líneas, el tamaño de parcela fue de un surco de las mismas dimensiones con 25 plantas. En Tlajomulco, el tamaño de parcela fue similar que en $\mathrm{Za}-$ popan, siendo las únicas diferencias, la longitud de la parcela, la cual se redujo a 4,0 m y en cada surco se dejaron 20 plantas. La densidad de población en ambas localidades fue de 62,500 plantas haI, aproximadamente.

En Zapopan los mestizos y sus líneas se sembraron el 16 de mayo de 1994 bajo condiciones de humedad residual con el manejo agronómico siguiente: se fertilizó con la dosis 184-92-00, distribuida en tres oportuni- 
dades, en la siembra se aplicó todo el fósforo, la mitad del nitrógeno en la etapa de décima a décima primer hoja ligulada (92-00-00) y el resto en prefloración (9200-00). Las plagas de la raíz se controlaron con una aplicación de $20 \mathrm{~kg}$ ha) de Carbofuran 5\% G mezclado con el fertilizante al momento de la siembra. Además, junto con el fósforo y el insecticida se mezclaron teliosporas de carbón de la espiga (Sphacelloteca reiliana) a razón de 2,0 kg ha) para inducir la enfermedad. No se controlaron insectos defoliadores debido a que las poblaciones fueron muy bajas. La maleza se controló con dos cultivos y como complemento, en el segundo cultivo se aplicaron cuatro litros de Metolaclor 25\% + Atrazina 23,5\% y un litro de Paraquat, diluidos en 200 litros de agua; además, se hicieron dos deshierbes manuales.

En Tlajomulco, los mestizos y sus líneas se sembraron bajo condiciones de temporal el 21 y 25 de junio, respectivamente. El manejo agronómico fue el siguiente: se fertilizó con la dosis 161-92-00 distribuida en dos oportunidades; en la siembra 69-92-00 y el resto (92-00-00) 30 días después. Las plagas de la raíz se controlaron con dos aplicaciones de $20 \mathrm{~kg} \mathrm{ha}^{-1}$ de Isosofos $5 \% \mathrm{G}$ en la siembra y en la segunda fertilización mezclados con el fertilizante. Los insectos defoliadores se controlaron con dos aplicaciones de $1 \mathrm{I} \mathrm{ha}^{-1}$ de Clorpirifos mezclado con dos litros de Byfolan +1 litro de Carbovit, para complementar la nutrición mineral del cultivo. La maleza se controló aplicando en preemergencia, por hectárea, la mezcla de tres litros terbutrina $25 \%$ + Atrazina 25\%, de 4 I Metolaclor 25\% + Atrazina $23,5 \%$ y dos litros de Paraquat, diluidos en 200 litros de agua, y se complementó con un deshierbe manual.

Las variables medidas fueron el rendimiento de grano, humedad a la cosecha, mazorcas por planta, floración masculina y femenina, altura de planta y ma- zorca, acame de raíz y tallo, mazorcas podridas y carbón de la espiga. Se hicieron análisis estadísticos por localidad para rendimiento de grano en mestizos y líneas y se obtuvo el promedio aritmético en el resto de variables. Con el promedio de entradas a través de localidades se estimaron correlaciones fenotípicas entre el rendimiento y otras características agronómicas dentro de mestizos, dentro de líneas, y entre mestizos y líneas, para todos los caracteres agronómicos.

\section{RESULTADOS Y DISCUSIÓN}

Los análisis de varianza detectaron diferencias estadísticas significativas para rendimiento de grano en los mestizos y en las líneas per se en las dos localidades de evaluación. Los 15 mestizos intermedios más sobresalientes rindieron $9,5 \%$ más que la media general y $24 \%$ más que el promedio de los testigos. El mestizo más rendidor (V-385 C3 x SI-265-2-2-1), superó en $15 \%$ al mejor testigo H-315 (Cuadro 1). En otros caracteres agronómicos, los 15 mestizos sobresalientes fueron similares a la media de los testigos en humedad a la cosecha, mazorcas/planta, precocidad y altura de planta y mazorca, pero tuvieron menor porcentaje de acame de raíz y pudriciones de la mazorca (Cuadro 1).

En el caso de los mestizos tardíos, la media de los 30 más rendidores superaron en $9,8 \%$ a la media general y en $22 \%$ a la media de los testigos, el mestizo más rendidor (V-385 $\left.\mathrm{C}_{3} \times \mathrm{ST}-35-1-8-3\right)$, fue $12 \%$ superior a la media del mejor testigo A-7520 (Cuadro 2). En promedio, los mestizos seleccionados tuvieron una altura de planta $3 \%$ mayor que la media de los testigos, pero los mestizos sobresalientes fueron más precoces y tuvieron menor acame de raíz y pudriciones de la mazorca (Cuadro 2) y que el probador tuvo rendimiento

Cuadro 1. Rendimiento de grano y características agronómicas de los mestizos de ciclo intermedio y testigos evaluados. Promedio de dos localidades.

\begin{tabular}{|c|c|c|c|c|c|c|c|c|c|c|c|}
\hline \multirow[t]{2}{*}{ Variable } & \multirow{2}{*}{$\begin{array}{l}\text { Rend. } \\
\text { (kg/ha) }\end{array}$} & \multirow{2}{*}{$\begin{array}{c}\mathrm{HC} \\
\%\end{array}$} & \multirow{2}{*}{$\begin{array}{l}\text { No. de } \\
\text { MPP }\end{array}$} & \multicolumn{2}{|c|}{ Días a flor. } & \multicolumn{2}{|c|}{ Altura $(\mathrm{cm})$} & \multicolumn{2}{|c|}{$\%$ de Acame } & \multirow{2}{*}{$\begin{array}{l}\text { MD } \\
(\%)\end{array}$} & \multirow{2}{*}{$\begin{array}{c}\mathbf{C E} \\
\%\end{array}$} \\
\hline & & & & Masc. & Fem. & Planta & Maz. & Raíz & Tallo & & \\
\hline \multicolumn{12}{|l|}{ Media general de } \\
\hline mestizos intermedios & 7221 & 15 & 0,96 & 69 & 71 & 244 & 113 & 15 & 2 & 9 & 0 \\
\hline \multicolumn{12}{|l|}{ Media de los quince } \\
\hline mestizos más rendidores & 7908 & 15 & 0,97 & 69 & 70 & 246 & 113 & 14 & 2 & 8 & 0 \\
\hline V-385 C3 $\times$ SI-265-2-2-1 & 8712 & 15 & 0,96 & 68 & 70 & 242 & 109 & 18 & 1 & 8 & 0 \\
\hline Media de testigos & 6354 & 14 & 0,88 & 69 & 71 & 242 & 107 & 18 & 1 & 11 & 0 \\
\hline H-315 & 7584 & 15 & 0,94 & 69 & 70 & 242 & 117 & 17 & 1 & 9 & 0 \\
\hline
\end{tabular}

$\mathrm{HC}=$ porcentaje de humedad a la cosecha.

$\mathrm{CE}=$ porcentaje de ataque de carbón de la espiga.
MPP = mazorcas por planta

$\mathrm{MD}=$ porcentaje de mazorcas completamente dañadas. 
Cuadro 2. Rendimiento de grano y características agronómicas de los mestizos de ciclo tardío y testigos evaluados. Promedio de dos localidades.

\begin{tabular}{|c|c|c|c|c|c|c|c|c|c|c|c|}
\hline \multirow[t]{2}{*}{ Variable } & \multirow{2}{*}{$\begin{array}{l}\text { Rend. } \\
\text { (kg/ha) }\end{array}$} & \multirow{2}{*}{$\begin{array}{l}\mathrm{HC} \\
\%\end{array}$} & \multirow{2}{*}{$\begin{array}{l}\text { No. de } \\
\text { MPP }\end{array}$} & \multicolumn{2}{|c|}{ Días a flor. } & \multicolumn{2}{|c|}{ Altura (cm) } & \multicolumn{2}{|c|}{$\%$ de Acame } & \multirow{2}{*}{$\begin{array}{l}\text { MD } \\
(\%)\end{array}$} & \multirow{2}{*}{$\begin{array}{l}\mathrm{CE} \\
\%\end{array}$} \\
\hline & & & & Masc. & Fem. & Planta & Maz. & Raíz & Tallo & & \\
\hline $\begin{array}{l}\text { Media general de } \\
\text { mestizos tardíos }\end{array}$ & 8001 & 17 & 1,01 & 71 & 72 & 255 & 121 & 12 & 2 & 8 & 0,3 \\
\hline Media de los treinta & & & & & & & & & & & \\
\hline mestizos más rendidores & 8782 & 16 & 1,04 & 70 & 72 & 263 & 124 & 12 & 2 & 7 & 0,2 \\
\hline V-385 C3 x ST-35-1-8-3 & 9933 & 14 & 1,17 & 71 & 71 & 251 & 120 & 5 & 2 & 8 & 0 \\
\hline Media de los testigos & 7223 & 15 & 0,98 & 72 & 74 & 256 & 126 & 15 & 3 & 11 & 0,2 \\
\hline A-7520 & 8879 & 15 & 0,96 & 69 & 70 & 264 & 124 & 10 & 5 & 10 & 0 \\
\hline V-385 C3 & 6376 & 17 & 0,93 & 70 & 72 & 243 & 111 & 29 & 5 & 14 & 2 \\
\hline
\end{tabular}

$\mathrm{HC}=$ porcentaje de humedad a la cosecha.

$\mathrm{CE}=$ porcentaje de ataque de carbón de la espiga.

MPP = mazorcas por planta.

$\mathrm{MD}=$ porcentaje de mazorcas completamente dañadas.

bajoy porcentajes altos de acame de raíz y tallo; tomando en cuenta que el programa de mejoramiento genético de maíz de la zona subtropical de México tiene como prioridad mejorar la resistencia al acame y el rendimiento de grano, estas características se consideran deseables en un probador, ya que de acuerdo con la teoría, el mejor probador es aquel que tiene frecuencias alélicas bajas para el caracter de interés (Hallauer y Miranda, 1988; Hallauer y López-Pérez, 1979; Rawlings y Thompson, 1962).

Es importante señalar, que aún cuando las medias generales de acame de raíz de los mestizos intermedios y tardíos fueron menores que el promedio de los testigos (Cuadros 1 y 2), sus valores se consideran todavía altos (15 y $12 \%$, respectivamente), por lo tanto la selección para resistencia al acame seguirá siendo prioritaria en el programa de mejoramiento genético de maíz de la zona subtropical de México.

Los bajos porcentajes de carbón de la espiga (Cuadros 1 y 2) se deben a que en Zapopan la fecha de siembra fue tardía y en dichas circunstancias se ha encontrado que se reduce la incidencia de la enfermedad (Ledezma, 1983).

Atendiendo el rendimiento de grano, en cada una de las Figuras 1 y 2 se presenta de manera gráfica la estrategia para seleccionar líneas en función de las medias de los mestizos (línea horizontal) y de las líneas per se (línea vertical), respectivamente. La mejor opción sería el grupo de líneas ubicadas en el cuadrante II, ya que conjuga altos rendimientos de las líneas per se y sus mestizos, lo cual favorecería la formación de híbridos, especialmente simples. Otra buena opción son las líneas del cuadrante II, pero con la desventaja de

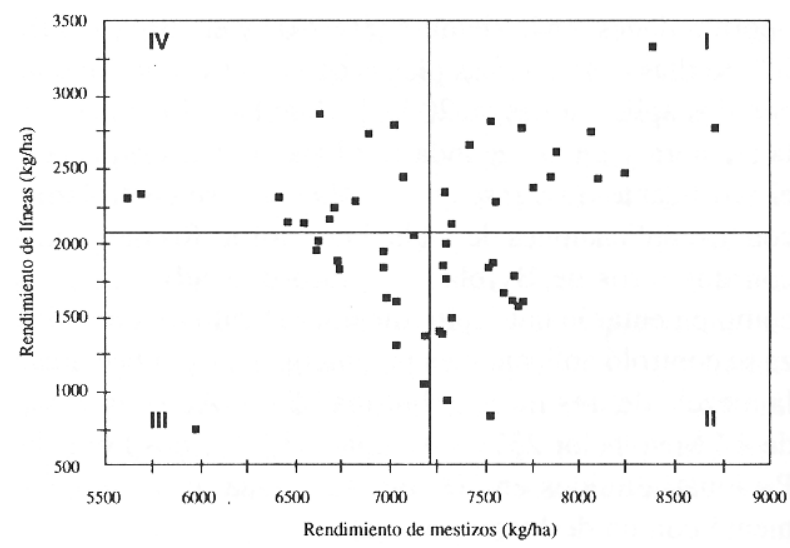

Figura 1. Rendimiento de grano ( $\mathrm{kg} / \mathrm{ha}$ ) de líneas y mestizos intermedios, promedio de dos localidades.

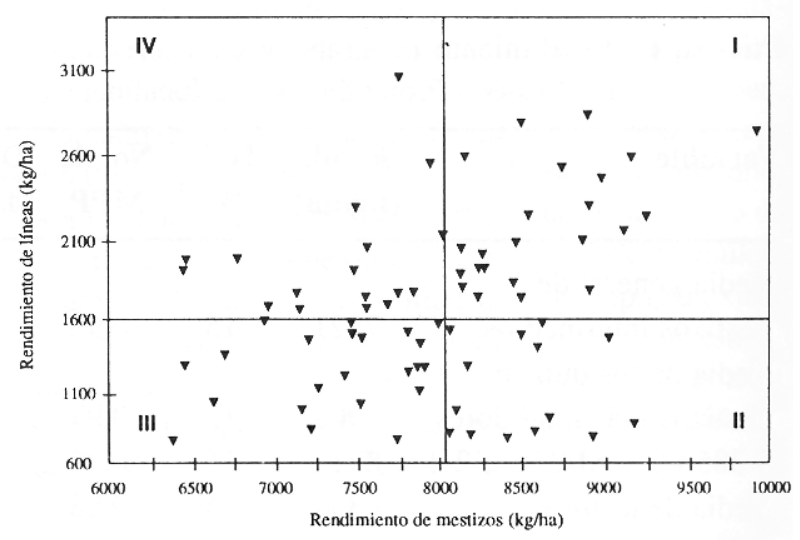

Figura 2. Rendimiento de grano (kg/ha) de líneas y mestizos tardíos, promedio de dos localidades. 
tener líneas de bajo rendimiento per se, aquí sólo sería costeable formar híbridos trilineales y dobles o simples modificados. No obstante, en la decisión final, deberán considerarse caracteres agronómicos de precocidad, resistencia al acame de raíz y tallo, pudriciones de mazorca, enfermedades, etc. Las líneas que deberán ser eliminadas son las de los cuadrantes III y IV. Nótese que sí la selección se hubiera hecho sólo con base en el rendimiento de la línea per se, se estarían escogiendo líneas con alto rendimiento per se y aptitud combinatoria general (ACG) negativa (cuadrante IV); además, se eliminarían líneas con ACG positiva (cuadrante 11).

Tomando en cuenta que la mayoría de caracteres de importancia económica son de herencia cuantitativa y por lo general tienen baja heredabilidad (Falconer, 1989), es deseable que antes de evaluar las líneas en mestizos, autofecundar al menos tres generaciones para incrementar la probabilidad de retener líneas superiores; ya que de acuerdo con Bernardo (1992) la correlación genética esperada entre líneas evaluadas en mestizos en $\mathrm{S}_{3}$ y $\mathrm{S}_{4}$ con respecto a la generación $\mathrm{S}$ ? fue de 0,94 y 0,97 , respectivamente. Estos resultados también ayudan a explicar porque los mejoradores de maíz de los Estados Unidos de América prefieren realizar la prueba tardía (Bauman, 1981).

Los coeficientes de correlación entre el rendimiento de grano y el resto de los caracteres agronómicos entre las líneas intermedias per se, fueron significativos para mazorcas/planta, floración masculina y femenina y porcentaje de mazorcas podridas; en cambio en los mestizos intermedios, la correlación sólo fue significativa para mazorcas podridas y porcentaje de ataque de carbón de la espiga (Cuadro 3). En el grupo de líneas tardías evaluadas per se, los coeficientes de correlación entre el rendimiento de grano y los caracteres agronómicos, fueron estadísticamente significativos para el número de mazorcas/planta, floración masculina y femenina, altura de planta y mazorca y porcentaje de mazorcas podridas; en cambio en los mestizos tardíos, el coeficiente de correlación sólo fue significativo para número de mazorcas/planta, altura de planta y mazorca, y porcentaje de mazorcas podridas (Cuadro 3).

Con base en los resultados anteriores se observa que la correlación entre el rendimiento de grano y los caracteres agronómicos varió de acuerdo al tipo de familia evaluada, siendo estas relaciones más altas para condiciones de homocigosidad, caso de líneas (familias de autohermanos), que de heterocigosidad, caso de mestizos (familias de medios hermanos); especialmente en los caracteres relacionados con el aspecto reproductivo como fueron: el número de mazorcas por planta y el número de días a floración masculina y femenina. Por lo tanto, la evaluación de las líneas per se permitió conocer los as-
Cuadro 3. Correlaciones fenotípicas entre el rendimiento de grano y otras características agronómicas en lineas de maíz de ciclo intermedio y de ciclo tardío evaluadas per se y en mestizos (línea x probador). Promedio de dos localidades.

\begin{tabular}{|c|c|c|c|c|}
\hline \multirow[t]{2}{*}{ Correlación } & \multicolumn{2}{|c|}{ Líneas intermedias } & \multicolumn{2}{|c|}{ Líneas Tardías } \\
\hline & Perse & Mestizo & Perse & Mestizo \\
\hline Rend : HC & $-0,04 \mathrm{NS}$ & $0,01 \mathrm{NS}$ & $-0,14 \mathrm{NS}$ & $-0,18 \mathrm{NS}$ \\
\hline “ “”” : MPP” & $0,49 * *$ & $0,15 \mathrm{NS}$ & $0,78 * *$ & $0,51 * *$ \\
\hline “ “"” : FM" & $-0,31 *$ & $-0,18 \mathrm{NS}$ & $-0,38 * *$ & $0,14 \mathrm{NS}$ \\
\hline “" “" : FF" & $-0,51 * *$ & $-0,17 \mathrm{NS}$ & $-0,50 * *$ & $0,03 \mathrm{NS}$ \\
\hline “ “" : APL" & $0,21 \mathrm{NS}$ & $0,08 \mathrm{NS}$ & $0,34 * *$ & $0,57 * *$ \\
\hline “ “" : AMZ” & $0,04 \mathrm{NS}$ & $0,05 \mathrm{~N}$ & $0,51 * *$ & $0,37 * *$ \\
\hline “ “"” : AR" & $-0,21 \mathrm{NS}$ & $0,04 \mathrm{NS}$ & $0,06 \mathrm{NS}$ & $0,01 \mathrm{NS}$ \\
\hline “" “"” : AT" & $0,00 \mathrm{NS}$ & $-0,10 \mathrm{NS}$ & $-0,10 \mathrm{NS}$ & $0,08 \mathrm{NS}$ \\
\hline “ “"”: MD” & $-0,61 * *$ & $-0,45 * *$ & $-0,49 * *$ & $-0,32 * *$ \\
\hline ““"”: : CE” & $0,02 \mathrm{NS}$ & $-0,35 * *$ & $-0,15 \mathrm{NS}$ & $-0,11 \mathrm{NS}$ \\
\hline
\end{tabular}

*, ** Significativo al 0,05 y 0,01

$\mathrm{APL}=$ altura de planta. $\mathrm{NS}=$ no significativo. $\mathrm{AMZ}=$ altura de mazorca. $\mathrm{HC}=$ porcentaje de humedad a la cosecha. $\mathrm{AR}=$ porcentaje de acame de raíz. $\mathrm{MPP}=$ número de mazorcas por planta. $\mathrm{AT}=$ porcentaje de acame de tallo. $\mathrm{FM}=$ número de días a floración masculina. $\mathrm{MD}=$ porcentaje de mazorcas podridas. $\mathrm{FF}=$ número de días a floración femenina. $\quad \mathrm{CE}=$ porcentaje de carbón de la espiga.

pectos agronómicos de las líneas que serán vitales en la producción de semillas y constituyó un complemento de la información de ACG obtenida en la evaluación de los mestizos; lo cual confirma la complementariedad señalada cuando fueron discutidas las Figuras 1 y 2.

Adicionalmente en las evaluaciones de líneas per se, se podría obtener información muy valiosa sobre otros caracteres importantes tales como: vigor, tipo de espiga, producción de polen, tipo de grano, y si las líneas fueran evaluadas en varios ambientes, sería posible estimar la estabilidad de los caracteres agronómicos, lo cual sería de suma importancia, considerando que las líneas normalmente han maniestado mayor interacción genotipo-ambiente que los mestizos (Luna et al., 1973; Galarza et al., 1973; González et al., 1990). En las correlaciones entre líneas y mestizos, todos los coeficientes de correlación fueron positivos y significativos, excepto para rendimiento de grano, porcentaje de mazorcas podridas y carbón de la espiga en el grupo tardío (Cuadro 4). Los coeficientes de correlación de rendimiento de grano en ambos grupos de líneas tuvieron valores similares y no significativos, a pesar de que las líneas evaluadas fueron derivadas de poblaciones 
Cuadro 4. Correlaciones fenotípicas de características agronómicas entre líneas de maíz evaluadas per se y sus mestizos correspondientes. Promedio de dos localidades. Campo experimental Centro de Jalisco. 1994.

\begin{tabular}{lcc}
\hline Correlación & $\begin{array}{c}\text { Líneas } \\
\text { Intermedias } \\
\text { Línea : Mestizo }\end{array}$ & $\begin{array}{c}\text { Líneas Tardías } \\
\text { Línea : Mestizo }\end{array}$ \\
\hline REND : REND & $0,21 \mathrm{NS}$ & $0,20 \mathrm{NS}$ \\
HC :HC & $0,62 * *$ & $0,54 * *$ \\
MPP : MPP & $0,55 * *$ & $0,25 *$ \\
FM :FM & $0,34 * *$ & $0,70 * *$ \\
FF : FF & $0,47 * *$ & $0,68 * *$ \\
APL : APL & $0,60 * *$ & $0,72 * *$ \\
AMZ : AMZ & $0,55 * *$ & $0,59 * *$ \\
AR : AR & $0,14 \mathrm{NS}$ & $0,31 * *$ \\
AT : AT & $0,58 * *$ & $0,52 * *$ \\
MD : MD & $0,17 \mathrm{NS}$ & $0,13 \mathrm{NS}$ \\
CE : CE & $0,56 * *$ & $0,15 \mathrm{NS}$ \\
\hline
\end{tabular}

$*, * *$ Significativo al 0,05 y 0,01

$\mathrm{NS}=$ no significativo.

$\mathrm{AR}=$ porcentaje de acame de raíz.

$\mathrm{AT}=$ porcentaje de acame de tallo.

$\mathrm{MD}=$ porcentaje de mazorcas podridas .

$\mathrm{CE}=$ porcentaje de carbón de la espiga.

$\mathrm{APL}=$ altura de planta

$\mathrm{AMZ}=$ altura de mazorca

$\mathrm{HC}=$ porcentaje de humedad a la cosecha.

MPP = número de mazorcas por planta.

FM = número de d'as a floración masculina

$\mathrm{FF}=$ número de días a floración femenina.

élite no emparentadas, lo cual confirma que no es posible inferir con base en el rendimiento de la línea per se sobre el rendimiento del mestizo y se plantee la estrategia de realizar ambas evaluaciones. En el caso del porcentaje de mazorcas podridas, la ausencia de correlación pudo haber surgido porque tanto las líneas como el probador se considera que tienen una frecuencia de alelos alta para sanidad de mazorca, puesto que han sido seleccionados para este caracter. El probador fue más eficiente para descriminar acame de tallo que de raíz, lo que significaría la presencia de una baja frecuencia de alelos en el mismo, para el primer caracter.

La estrategia de efectuar la prueba tardía para ACG, evaluando líneas per se y mestizos, simultáneamente; tiene como desventaja que el costo de la evaluación de líneas se eleva, debido a los gastos adicionales de multiplicación de líneas y su evaluación per se en ensayos repetidos a través de ambientes. Sin embargo, esta desventaja se compensaría ampliamente debido que en las primeras tres o cuatro generaciones de autofecundación las líneas se seleccionarían para caracteres agronómicos, lo que da oportunidad a incrementar la calidad de las mismas y como consecuencia a disminuir considerablemente el número de ellas, reduciéndose los costos de evaluación. Asimismo, permitiría a los mejoradores definir anticipadamente el tipo de híbrido a formar, y el papel (hembra o macho) que jugarían los progenitores seleccionados en la formación de híbrido s comerciales.

Por último, otro aspecto importante a considerar en esta estrategia, es que en las evaluaciones de líneas per se se deberían conducir con un excelente manejo agronómico para reducir los problemas de interacción genotipo-ambiente en la selección de líneas.

\section{CONCLUSIONES}

El uso de la prueba tardía $\left(\mathrm{S}_{3}\right.$ ó $\mathrm{S}_{4}$ en adelante), la evaluación simultánea de líneas per se y el método gráfico desarrollado, es una estrategia importante para seleccionar líneas con alta aptitud combinatoria general y calidad agronómica. Además, es útil para reducir el número de líneas a evaluar y a orientar anticipadamente el tipo de híbrido a formar.

La no significancia y similitud del coeficiente de correlación entre el rendimiento de grano de las líneas per se y los mestizos, en ambos grupos de líneas $(r=0,21$ y $\mathrm{r}=0,20$ para líneas intermedias y tardías, respectivamente), confmna que no es del todo confiable seleccionar líneas con base en el rendimiento de sus mestizos, 1 o cual justifica la estrategia de realizar ambas evaluaciones.

\section{LITERATURA CITADA}

BAUMAN, L.E 1981. Review of methods used by breeders to develop superior inbreds. Proc. Corn and Sorghum Ind. Res. Conf.. 36: 199-208.

BERNARDO, R. 1992. Retention of genetically superior lines during early generations testcrossing of maize. Crop Sci. 32: 933-937.

FALCONER, D.S. 1989. Introduction to quantitative genetics. Third Edition. John Wiley \& Sons, Inc., New Yord. $437 \mathrm{p}$.

GALARZA S., M.; ANGELES A., H. H.; MOLINA GALÁNL, J, 1973. Estudio comparativo entre la prueba de líneas per se y la de mestizos para evaluar aptitud 
combinatoria general de líneas $\mathrm{S}_{1}$ de maíz (Zea mays L.). Agrociencia 11: 127-139.

GARCÍA, E. 1973. Modificaciones al sistema de clasificación climática de Köppen. Instituto de Geografía. UNAM.

GONZÁLEZ G. J.; MOLINA G., 1. D.; MARTÍNEZ G., A 1990. Implicación del rendimiento per se y de la ACG de líneas autofecundadas de maíz (Zea mays L.) en la producción de cruzas simples de alto rendimiento. Agrocienda Serie Fitociencia 2:29-42.

HALLA UER, A.R. 1990. Methods used in developing maize inbreds. Maydica. 36 1-19.

HALLAUER, AR.; LOPEZ-PEREZ, E. 1979. Comparison among testers for evaluating lines of corno Proc. Corn and Sorghum Ind. Res. Conf. 34: 57- 75.

HALLAUER, AR.; MIRANDA FO, J.B. 1988. Quantitative genetics in maize breeding. 2nd ed. lowa State Univ.

Press. Ames, IA.

HORNER, E.S.; CHAPMAN, W.H.; LUTRICK, M.C.; LUNDY, H.W. 1969. Comparison of selection based of yield of topcross progenies and of $\mathrm{S} 2$ progenies in maize (Zea mays L.). Crop Sci. 9:539-543.

JENKINS, M.T. 1978. Maize breeding during the development of early years of hybrid maize. In Maize breeding and genetics. Ed. B. D. Walden. John wiley \& Sons. New York. pp. 13-27.
JENKINS, M.T. 1935. The effect of inbreeding and of selection within inbred lines of maize upon the hybrids made in successive generations of selfing. lowa State Coll. J. Sci. 9: 429-450.

LEDEZMA M., J. 1983. Estudios sobre el control del carbón de la espiga del maíz Sphacellotheca reiliana (Kuhn Clinton) en el Valle de Zapopan, Jal. Tesis de Lic. Facultad de Ciencias. Universidad Autónoma de México. 67 p.

LONNQUIST, 1.H. 1968. Further evidence on testcross vs lines performance in maize. Crop Sci. 8: 50-53.

LUNA E, M.; MOLINA G., J.; ANGELES A, H. 1973. Comparación de métodos para evaluar aptitud combinatoria general de líneas de maíz (Zea mays L.) en relación al tamaño de muestra del probador. Agrociencia 11: $29-41$.

MÁRQUEZ S., E 1985. Genotecnia Vegetal. Tomo I. Métodos, teoría y resultados. AGT Editor, S.A. México, D. E $356 \mathrm{p}$.

RAWLINGS, J. O.; THOMPSON, D. L. 1962. Performance level as criterion of for the choice of maize testers. Crop Sci. 2: 217-220.

SPRAGUE, G. E 1946. Early testing of inbred lines of corno Jour. Amer. Soc. Agron. 38: 108-117.

SPRAGUE, G. E; TATUM, L. A. 1942. General vs specific combining ability in single crosses of corno Jour. Amer. Soc. Agron. 34: 923-932. 
